\title{
CPD QUESTIONNAIRE
}

\section{Vol. 13, No. 3}

Two CPD points are awarded for the correct completion and submission of the questions below.

CPD questionnaires must be completed online via www.cpdjournals.co.za. After submission, you can check the answers and print your certificate.

This programme is available free of charge to members of the HIV Clinicians Society and SAMA only.

TRUE (A) or FALSE (B) - click on the correct answer:

Regarding Addison's disease in HIV-infected individuals: 1. HIV has a direct effect on the adrenal glands and may also disrupt the hypothalamic-pituitary-adrenal axis.

2. CT scanning is not a valuable investigative modality in suspected Addison's disease in an HIV-infected individual.

\section{Regarding non-progression of HIV disease:}

3. Long-term non-progressors (LNTPs) are defined as individuals who take longer than 10 years to progress to AIDS, while elite controllers are individuals who maintain relatively low levels of viraemia without antiretroviral therapy (ART).

4. LNTPs and elite controllers are determined by host genetics and HIV subtype; viral diversity has little role in determining which individuals will be LTNPs.

5. There is strong evidence that all LNTPs and elite controllers will benefit from immediate initiation of ART.

Regarding management of depression in individuals receiving ART:

6. Treating depression can improve ART adherence, and antidepressive medication is superior to psychotherapeutic interventions for this purpose.

Regarding choice of antiretroviral drugs for adult therapy: 7. Abacavir $(\mathrm{ABC})$ hypersensitivity occurs in $10 \%$ of African patients, and this increased frequency is the principal reason why this agent is not widely recommended in South African guidelines.

8. In adults, $\mathrm{ABC}$ is commonly used in single-drug substitutions required due to toxicity to specific agents (e.g. peripheral neuropathy attributed to $\mathrm{D} 4 \mathrm{~T}$ ).

9. Protease inhibitors (PIs) can cause dyslipidaemia; as a result, a statin should be routinely considered in all HIV-infected patients starting lopinavir/ritonavir.
10. Among NRTIs, zidovudine is more likely to cause hyperlactataemia than lamivudine.

11. In the event of mild ART toxicity, it is advisable to stop all antiretroviral agents, and re-introduce one agent at a time to identify (and then avoid) the responsible agent.

12. The combination of TDF and ddI should be avoided due to poor virological outcomes.

13. There is evidence to suggest that $A B C$ may not be as effective as tenofovir in achieving and sustaining viral suppression in adults.

14. ART failure is best defined as two viral load measures $>400$ copies $/ \mathrm{ml}$ on specimens approximately 2 weeks apart.

15. In HIV-infected patients who also have hepatitis B, TDF and 3TC play an important role in managing both infections.

16. Saquinavir, if affordable, is the PI of choice in individuals with no prior PI exposure.

17. The use of two NRTIs should be avoided in second-line therapy, if at all possible.

18. Emtricitabine and lamivudine are essentially interchangeable in terms of their efficacy.

19. Gynaecomastia is a notable side-effect of efavirenz.

Regarding integration of tuberculosis (TB) services into HIV care and treatment:

20. Evidence from HIV clinics in Gauteng suggests that isoniazid preventive therapy and $\mathrm{TB}$ screening are being implemented for the vast majority of patients, thereby helping to reduce the burden of TB in HIV-infected individuals.

\section{INSTRUCTIONS}

1. Read the journal. All the answers will be found there.

2. Go to www.cpdjournals.co.za to answer the questions.

Accreditation number: MDB001/028/10/2011 (Clinical) 


\section{The Power of 3 The Simplicity of 1}

\section{tribuss $^{\text {TM }}$}

Tenofovir DF $300 \mathrm{mg}$ Emtricitabine $200 \mathrm{mg}$

Efavirenz $600 \mathrm{mg}$

\section{Once Daily Fixed Dose Combination}

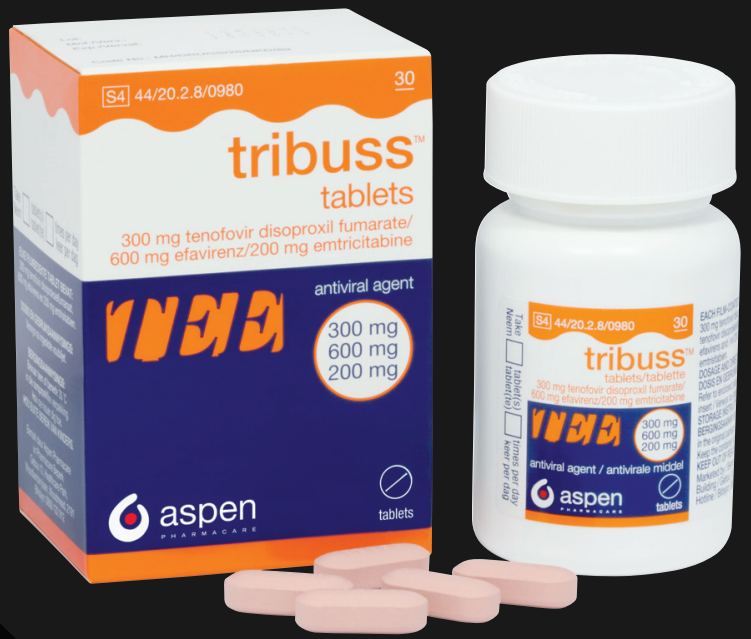

○ aspen Healthcare. We Care.

Marketed by Aspen Pharmacare www.aspenpharma.com Medical Hotline 0800118088
S4 Tribuss ${ }^{\top M}$. Reg. No: 44/20.2.8/0980. Each film coated tablet contains $300 \mathrm{mg}$ tenofovir disoproxil fumarate, $200 \mathrm{mg}$ emtricitabine and $600 \mathrm{mg}$ efavirenz. For full prescribing informatic prescribing information, refer to the p regulatory authority. Applicant: Pharmacare Limited. Co. Reg. No.. 1898/000252/06. Building 12, Healthcare Park, Woodlands Drive, Woodm Fax (011) 239 3438. A13853 11/11. 